Monheim LM. Analgesia in dentistry now and in the future. Anesth Prog. 1971;18(5):100-106 and J Am Analg Soc. 1971:9:1(abstract).

The evolution and history of dental sedation and anesthesia offers perspectives and insights on our present practice. This commentary, honoring Dr Joel Weaver, has additionally and uniquely been made possible through the labors of Joel and Barbara Weaver. They scanned decades worth of Anesthesia Progress content into the now readily available archives for future generations seeking this background information, which represents only a small fraction of Dr Weaver's legacy in dental anesthesia.

Upon reviewing these archives, the paper by Dr Leonard Monheim, "Analgesia in Dentistry Now and in the Future," stands out as being a seminal treatise on the concept of conscious sedation, or what is now termed moderate sedation. Dr Monheim was a driving force in setting standards for sedation and anesthesia in dentistry. His influence at the University of Pittsburgh, on his distinguished successors, and on the dental profession is legend. The fundamental concepts that comprise conscious sedation for dentistry, initially established during the mid-20th century, have undergone substantial development, particularly over the last 4 decades. Previously, dental anesthesia was confined to the realm of unconscious techniques, primarily for short oral surgical procedures. Dr Monheim and his contemporaries throughout the United States and abroad worked to develop techniques that could be used safely in the office by dentists for longer procedures such as restorative dentistry.

As mentioned within Dr Monheim's article, definitions play an integral part in our professional lives. They impact criteria for sedation and general anesthesia state permitting requirements and influence malpractice carrier standards and insurance reimbursement. Today, the Commission on Dental Accreditation develops training requirements for all US dental students, general dentists, and dental specialties in the areas of sedation and general anesthesia. Pioneers like $\mathrm{Dr}$ Monheim worked to appropriately define sedation, recognizing the importance of having standardized descriptions of the changes that occur throughout the sedation continuum. As we see from Dr Monheim's discussion, accurate and logical definitions were previously lacking. This was akin to many of the various statements made as the country attempted to define obscenity in the 1960s.

Anesth Prog 66:232-234 2019 | DOI 10.2344/anpr-66-04-09

(C) 2019 by the American Dental Society of Anesthesiology
"I shall not today attempt further to define the kinds of material I understand to be embraced within that shorthand description and perhaps I could never succeed in intelligibly doing so. But $I$ know it when $I$ see it. ..." Associate Justice Potter Stewart

Until Dr Monheim's presentation and publication, it was, "I know it when I see it," without any foundation in scientific or evidence-based medicine. Dr Monheim begins by bemoaning the lack of proper and acceptable nomenclature. How could there be a relevant scientific discussion if terms such as analgesia, intravenous analgesia, intravenous anesthesia, intravenous sedation, chemanesia (a term first used by Monheim himself), chemalgesia, hypoesthesia, hypoalgesia, and neuroleptanalgesia were all being bandied about? Additionally, terms such as sedoamnesia, lytic cocktails, twilight sleep, and relative analgesia were heard in lectures and seen in publications. If there is not agreement on what to call this, how can it be determined as to who should professionally perform it, what training should be required, what drugs should be used, and what auxiliary personnel should be present? To bring order to this chaos, Dr Monheim suggested that the term "sedalgesia" be used in lieu of all others. He states that sedalgesia would accurately describe how the use of sedation and analgesia would act to lessen the impact of an unpleasant situation upon a conscious patient. That statement is the very crux of what Monheim's student and successor, Dr C. Richard Bennett, termed conscious-sedation in his classic text, Conscious-Sedation in Dental Practice. Dr Monheim wanted to make it clear that there must be a definite distinction between sedalgesia and general anesthesia. He stressed that either the patients were conscious or they were not! This distinction was important because it not only spoke to patient safety but also helped determine the level of training required to perform it.

With the passing of time, the term sedalgesia never caught on. Conscious sedation became the universally accepted terminology for many years. Now, in our profession's need to further delineate patient management, the terms minimal sedation, moderate sedation, deep sedation, and general anesthesia are currently in use. In an attempt to describe minute differences in the level of consciousness, for example, can the patient respond to light tactile stimulation or only to a deep painful one, these new terms may have again muddied the nomenclature arena. The gold standard continues to be either the patient is conscious or not.

Monheim realized that although sedalgesia was designed to maintain patient consciousness and cooperation plus raise the pain threshold, regional analgesia 
was necessary to fully block pain impulses. A profound nerve block was essential to the success of his conscious technique, because only general anesthesia reliably blocks all pain stimuli. He discusses the dual nature of pain, stressing that pain must not only be perceived but also appreciated as such. This is the dividing line between what is now called moderate sedation and deep sedation. He believed, as we do now, that intentionally crossing this plane must only be accomplished by those practitioners with advanced airway management skills and general anesthesia training.

Altering the patient's mood while maintaining consciousness and protective reflexes was Dr Monheim's vision for the future of sedation in dentistry. He believed that if one could follow the principles of sedalgesia, then any dentist could master the technique if they so desired. He believed strongly in a universal sedation curriculum in predoctoral dental education. He so believed that dental students had the educational background to learn his techniques that he began teaching third- and fourthyear students to use sedalgesia at the University of Pittsburgh, as attested by Drs Giovannitti and Phero, who took this elective as dental students in the 70s. This tradition in education has been carried out to this day in Pittsburgh. In fact, it was involvement in this elective as predoctoral students that led many to graduate residencies in dental anesthesiology.

Aside from specific drugs, it is interesting how little the pharmacologic principles discussed by Dr Monheim have changed in the last 50 years. The sedalgesia technique was based upon the use of 3 drug classes, given either individually or more commonly in combination. The key was their administration in titrated incremental divided doses in order to observe the patient's response, thus stopping short of producing unconsciousness. Narcotics (opioids) were used to raise the pain threshold and alter the mood. A new psychoactive drug, diazepam, was found to be more effective than previously available psychosedative drugs, barbiturates and phenothiazine derivatives, due to its ability to produce a remarkable calming effect and amnesia. In small doses, the ultra-short-acting barbiturates were used to produce changes in mood. The same general technique is used today, albeit with newer drugs that offer greater degrees of control. Fentanyl is currently the most widely used opioid for moderate sedation, and midazolam has supplanted diazepam as the benzodiazepine of choice. Propofol has replaced the barbiturates, and a new class of alpha-2 agonists (dexmedetomidine) has shown great promise for moderate sedation. It is interesting that $\mathrm{Dr}$ Monheim dismissed ketamine as being inappropriate for his sedalgesia technique due to its inconsistency of action, prolonged recovery, and propensity for psychotic reactions. Today, ketamine is still not considered to be an appropriate choice for moderate sedation providers.

Dr Monheim's belief in education motivated him to advocate for universal educational guidelines to standardize the training and use of sedation for all dentists. The American Dental Association formally accepted the first guidelines regarding the teaching and use of pain control, sedation, and anesthesia in 1971, shortly after his death. Since then, advanced education programs in dental anesthesiology must conform to Commission on Dental Accreditation standards that ensure consistency of training. Graduates of these programs are examined and certified by the American Dental Board of Anesthesiology and are required to maintain certification through continual postgraduate education. Dr Monheim and Dr Harry Archer (Chair of the Department of Oral Surgery in Pittsburgh) had a vision to establish the first independent department of anesthesiology in a dental school in 1949. At the University of Pittsburgh, as an example, all dental students are trained in local anesthesia, nitrous oxide, enteral sedation, and clinical medicine. Training in intravenous moderate sedation is offered on an elective basis, and the 3-year anesthesiology residency program for dentists is ongoing. Dr Monheim's model has resulted in a faculty of 4 full-time and 6 part-time dentist anesthesiologists that maintain a large clinical presence within the school, further optimizing patient care and safety.

Although there are several other schools with dentist anesthesiologists providing a multitude of services including residency training programs, Monheim's vision for the future has not yet been fully met. Dental education has not completely embraced dental anesthesiology for reasons unknown. Perhaps it has been the earlier lack of specialty recognition and/or the lack of appreciation of the value of anesthesia education within a predoctoral curriculum. In programs around the country, experience demonstrates that students receiving advanced anesthesia education become practitioners that now safely and consistently meet the spectrum of patient care needs. They have the ability to fully assess a patient's medical condition and safely incorporate sedation modalities. It is our hope that with specialty recognition of anesthesiology in dentistry, dental students will begin to question why dental anesthesia may not be represented at their schools and dental deans will come to realize the value of a comprehensive predoctoral sedation curriculum. Dr Monheim was accurate when stating, "The future of sedalgesia lies in better education for the student and a more sincere dedication from the practitioner." (p. 106). 
Joseph A. Giovannitti, DMD

*Professor and Chair, Department of Dental

Anesthesiology

University of Pittsburgh School of Dental Medicine

Pittsburgh, Pennsylvania

Morton B. Rosenberg, DMD

$\dagger$ Professor Emeritus, Department of Oral and

Maxillofacial Surgery

Tufts University School of Dental Medicine

Professor of Anesthesiology and Perioperative Medicine

Tufts University School of Medicine

Boston, Massachusetts

James C. Phero, DMD

$\ddagger$ Professor Emeritus, Anesthesiology

University of Cincinnati College of Medicine

Cincinnati, Ohio

\section{CONTINUING EDUCATION QUESTIONS}

This continuing education (CE) program is designed for dentists who desire to advance their understanding of pain and anxiety control in clinical practice. After reading the designated article, the participant should be able to evaluate and utilize the information appropriately in providing patient care.

The American Dental Society of Anesthesiology (ADSA) is accredited by the American Dental Association and Academy of General Dentistry to sponsor CE for dentists and will award CE credit for each article completed. You must answer 3 of the 4 questions correctly to receive credit.

\section{Submit your answers online at www.adsahome.org. Click on "On Demand CE."}

$\mathrm{CE}$ questions must be completed within 3 months and prior to the next issue.

1. All of the following are criteria necessary for establishing the "sedalgesic state" as discussed by Dr. Monheim EXCEPT:

a. the patient should be amnestic and completely unconscious.

b. the patient should be awake and cooperative.

c. the patient's pain threshold should be elevated.

d. the patient's protective reflexes should be active and intact.

2. According to Dr. Monheim, the prime drug to be used for "sedalgesia" to maximize safety and controllability is:
a. ketamine.
b. methoxyflurane.
c. morphine.
d. nitrous oxide.

3. Dr. Monheim mentions several considerations when using diazepam for "sedalgesia" including the fact that:

a. it causes profound persistent hypotension.

b. it has a wide margin of safety.

c. it ideally is used without local anesthesia.

d. it is incapable of causing unconsciousness.

4. As discussed by Dr. Monheim and the authors of the current commentary, the safe and effective use of moderate sedation during invasive dental procedures includes all of the following critical aspects EXCEPT:
a. careful titration of drugs to a desired endpoint.
b. ensuring profound local or regional anesthesia.
c. ensuring unconsciousness to guarantee amnesia.
d. restricting solid foods and liquids preoperatively. 